\title{
A note on Gröbner bases and graph colorings
}

\author{
Amir Hashemi and Zahra Ghaeli
}




\title{
A NOTE ON GRÖBNER BASES AND GRAPH COLORINGS
}

\author{
AMIR HASHEMI AND ZAHRA GHAELI
}

Received October 21, 2011

\begin{abstract}
In this paper, we correct a minor misstatement in [4], where J.A. De Loera demonstrates an explicit universal Gröbner basis of the radical ideal of a variety related to chromatic numbers. We show that this result does not hold when the base field is finite, and we correct it for this case.
\end{abstract}

2000 Mathematics Subject Classification: 13P10; 05C15

Keywords: graph coloring, chromatic numbers, Gröbner bases

\section{INTRODUCTION}

The theory of Gröbner bases is a key computational tool for studying polynomial ideals. This theory has been introduced and developed by Buchberger in 1965 (see his $\mathrm{PhD}$ thesis [2]) and has been applied to the problem of graph coloring in [1]. A graph with $n$ vertices may be represented by a polynomial in $n$ variables. This polynomial lies in a particular ideal if and only if the graph is not $k$-colorable. Thus, the problem of $k$-coloring a graph is equivalent to an ideal membership problem. The concept of Gröbner bases may be applied to solve this problem. It has been shown in [4] that the Gröbner basis of the ideal corresponding to this problem is universal, i.e. it is a Gröbner basis for any monomial ordering.

Let $k, n \geq 2$ be two positive integers and $K$ be an arbitrary field. Let $V(n, k)$ denote the set of vectors which have at most $k-1$ distinct coordinates. Let also $J(n, k)$ be the vanishing ideal of $V(n, k)$. De Loera in [4] has proved the following theorem:

Theorem 1. The set of polynomials

$$
\rho(n, k)=\left\{\prod_{1 \leq r<s \leq k}\left(x_{i_{r}}-x_{i_{s}}\right) \mid 1 \leq i_{1}<\cdots<i_{k} \leq n\right\}
$$

is a universal Gröbner basis for $J(n, k)$.

To prove this result, De Loera in his paper, on page 3, states that "... but no nonzero univariate polynomial belongs to $J(n, 2)$." However, this claim (and thus this 
theorem) holds only if $K$ is infinite. In the following example, we show that Theorem 1 fails when $K$ is a finite field.

Example 1. Let $K=\mathbb{F}_{2}=\{0,1\}$ be a field with two elements. Then $V(2,2)=$ $\{(0,0),(1,1)\}$, and therefore $x_{1}^{2}-x_{1}$ and $x_{2}^{2}-x_{2}$ belong to the ideal $J(2,2) \subset \mathbb{F}_{2}\left[x_{1}, x_{2}\right]$. From the above notations, we have $\rho(2,2)=\left\{x_{1}-x_{2}\right\}$ which is not a universal Gröbner basis for $J(2,2)$.

Extending Theorem 1 to the finite fields we prove the following theorem:

Theorem 2. Let $q=p^{e}$ where $p$ is a prime and e is a positive integer. Let also $K=\mathbb{F}_{q}$ be a finite field with $q$ elements. Then, the set of polynomials

$$
\tau(n, k)=\left\{\prod_{1 \leq r<s \leq k}\left(x_{i_{r}}-x_{i_{s}}\right) \mid 1 \leq i_{1}<\cdots<i_{k} \leq n\right\} \cup\left\{x_{i}^{q}-x_{i} \mid 1 \leq i \leq n\right\}
$$

is a universal Gröbner basis for $J(n, k)$.

It is worth commenting that for the applications of Theorem 1 in [4], De Loera has used this theorem for infinite fields. Now, we give the structure of the paper. In Section 2 we prove Theorem 2. Section 3 is devoted to a correction of Example 3.4 in [4] on enumerating distinct colorings.

\section{THE PROOF OF THEOREM 2}

In this section, we prove Theorem 2, using the proof structure of Theorem 1 in [4]. We briefly state some necessary definitions.

Let $q=p^{e}$ where $p$ is a prime and $e$ is a positive integer. Let $K=\mathbb{F}_{q}$ be a finite field with $q$ elements, $R=K\left[x_{1}, \ldots, x_{n}\right]$ be a polynomial ring and $I=\left\langle f_{1}, \ldots, f_{t}\right\rangle$ be the ideal of $R$ generated by polynomials $f_{1}, \ldots, f_{t}$. Let $f \in R$ and $\prec$ be a monomial ordering on $R$. The leading monomial of $f$ is the greatest monomial (with respect to $\prec$ ) which appears in $f$, and we denote it by $\operatorname{LM}(f)$. The leading coefficient of $f$, written $\operatorname{LC}(f)$, is the coefficient of $\operatorname{LM}(f)$ in $f$. The leading term of $f$ is $\mathrm{LT}(f)=\operatorname{LC}(f) \operatorname{LM}(f)$. The leading term ideal of $I$ is defined as

$$
\operatorname{LT}(I)=\langle\operatorname{LT}(f) \mid f \in I\rangle .
$$

For a finite set $G \subset R$, we denote by $\operatorname{LT}(G)$ the monomial ideal $\langle\operatorname{LT}(g) \mid g \in G\rangle$. A finite subset of polynomials $G \subset I$ is called a Gröbner basis for $I$ w.r.t. $\prec$ if $\mathrm{LT}(I)=\mathrm{LT}(G)$, see [3] for more details. A universal Gröbner basis for $I$ is a finite subset of $I$ which is a Gröbner basis w.r.t. any monomial ordering.

Proof of Theorem 2. The following lemma gives a set of conditions for a universal Gröbner basis ([4], Lemma 2.1). 
Lemma 1. Let $I \subset R$ be an ideal and let $G=\left\{g_{1}, \ldots, g_{t}\right\} \subset I$ be a set of polynomials such that each $g_{i}$ is a product of linear factors in $x_{1}, \ldots, x_{n}$. Further, assume that for any $g \in G$ and for any permutation $\sigma$ on the set $\{1, \ldots, n\}$, we have $g\left(\sigma\left(x_{1}\right), \ldots, \sigma\left(x_{n}\right)\right) \in G$. If $G$ is a Gröbner basis for I w.r.t. a particular monomial ordering, then it is a universal Gröbner basis for $I$.

In order to apply Lemma 1 to $\tau(n, k)$, we must prove the following three claims:

- Any $g \in \tau(n, k)$ factors into linear factors in $R$

- $g\left(\sigma\left(x_{1}\right), \ldots, \sigma\left(x_{n}\right)\right) \in \tau$ for each $g \in \tau(n, k)$ and any permutation $\sigma$ on the set $\{1, \ldots, n\}$

- $\tau(n, k)$ is a Gröbner basis for $J(n, k)$ w.r.t. a particular monomial ordering.

For the first item, it is enough to prove that $x_{i}^{k}-x_{i}$ for any $i$ factors into linear factors. This is deduced from the following lemma (see [6], Lemma 2.4).

Lemma 2. With the above notations, the polynomial $x^{q}-x$ factors (into linear factors) in $K[x]$ as

$$
x^{q}-x=\prod_{a \in K}(x-a) .
$$

The second item follows from the structure of $\tau(n, k)$, and the fact that the elements of $\rho(n, k)$ are in bijection with the $k$ element subsets of $\left\{x_{1}, \ldots, x_{n}\right\}$. Now, we deal with the third item. We prove that $\tau(n, k)$ is a Gröbner basis for $J(n, k)$ w.r.t. the lexicographical ordering $\prec$ with $x_{n} \prec \cdots \prec x_{1}$. Since $\tau(n, k) \subset J(n, k)$, it is enough to prove that the leading term of any polynomial in $J(n, k)$ is divisible by the leading term of a member of $\tau(n, k)$. For this, we proceed by a double induction on $k$ and $n$ like in the proof of Theorem 1 in [4]. Let $k=2$ and $n$ arbitrary. We know that

$$
\tau(n, 2)=\left\{x_{i}-x_{j} \mid 1 \leq i<j \leq n\right\} \cup\left\{x_{i}^{q}-x_{i} \mid 1 \leq i \leq n\right\}
$$

and $\operatorname{LT}(\tau(n, 2))=\left\{x_{1}, \ldots, x_{n-1}, x_{n}^{q}\right\}$. Let $f \in J(n, 2)$ be a nonzero polynomial. If $\mathrm{LT}(f)$ is divisible by any of the first $n-1$ variables then $\mathrm{LT}(f) \in \mathrm{LT}(\tau(n, 2))$. Otherwise, $f$ is a nonzero univariate polynomial in $x_{n}$ (we consider it in $K\left[x_{n}\right]$ ). From the definition of $V(n, 2)$ we can conclude that $f(a)=0$ for any $a \in K$. This implies that $x_{n}^{q}-x_{n}$ divides $f$, and therefore $x_{n}^{q}$ divides $\operatorname{LT}(f)$. By induction on $k$ the result is true for $J(n, r)$ for where $k>r \geq 2$ and $n$ arbitrary. We proceed by induction on $n$. We show that $J(k, k)$ is generated by the set $\left\{\prod_{1 \leq i \leq j \leq k}\left(x_{i}-x_{j}\right), x_{1}^{q}-x_{1}, \ldots, x_{k}^{q}-\right.$ $\left.x_{k}\right\}$. By Buchberger criterion and Buchberger first criterion (see [3], pages 85 and 104) we can prove easily that the set $B=\left\{x_{1}^{q}-x_{1}, \ldots, x_{k}^{q}-x_{k}\right\}$ is a Gröbner basis for the ideal that it generates. Let $f$ be an element of $J(k, k)$ and $\bar{f}$ be the remainder of the division of $f$ by $B$. It is worth noting that since $B$ is a Gröbner basis, this remainder is unique (see [3], Proposition 1 page 82). Since $\bar{f} \in J(k, k)$, regardless of whether $\bar{f}$ is zero or non-zero $\bar{f}\left(a_{1}, \ldots, a_{k}\right)=0$ for any $\left(a_{1}, \ldots, a_{k}\right) \in V(k, k)$. In $V(k, k)$, every $k$-dimensional point has at most $k-1$ distinct entries. Thus, if any two entries (such as $x_{i}$ and $x_{j}$ ) are equal, even if the other $k-2$ entries are distinct, 
the point is still contained in $V(k, k)$. Therefore, since $\bar{f}$ vanishes on every point in $V(k, k)$ then we have $\left(x_{i}-x_{j}\right) \mid \bar{f}$ for $1 \leq i<j \leq k$, and $\bar{f} \in \tau(k, k)$. This settles the case $n=k$.

Now by induction hypothesis the result is true for $J(r, k)$ with $n>r \geq k$. We have to prove that $\operatorname{LT}(f) \in \operatorname{LT}(\tau(n, k))$ for any $f \in J(n, k)$. Let $B=\left\{x_{1}^{q}-x_{1}, \ldots, x_{n}^{q}-\right.$ $\left.x_{n}\right\}$, which is a Gröbner basis for the ideal that it generates. Let $\bar{f}$ be the remainder of the division of $f$ by $B$. We have $\bar{f} \in J(n, k)$. If $\bar{f} \neq 0$, we construct an auxiliary polynomial. Let $S \subseteq\{1, \ldots, n-1\}$. We denote by $\bar{f}_{S}$ the polynomial obtained from $\bar{f}$ by substituting $x_{n}$ for each variable $x_{i}$ for $i \in S$. Thus, for a non-empty set $S$ the polynomial $\bar{f}_{S} \in J(r, k)$ with $r=n-|S|$ where $|S|$ denotes the size of $S$. Let

$$
g=\sum_{S \subseteq\{1, \ldots, n-1\}}(-1)^{|S|} \bar{f}_{S} .
$$

We claim that $\operatorname{LT}(g) \in \operatorname{LT}(\tau(n, k))$. Note that from the definition of $\bar{f}$ we can replace $\tau(n, k)$ by $\rho(n, k)$ in this claim. The rest of the proof is exactly the same as the latter part of the proof of Theorem 1 in [4]. However, for the sake of completeness, we provide it here. If we substitute $x_{n}$ for any $x_{i}$ with $1 \leq i \leq n-1$ then we get the zero polynomial (note that $\operatorname{deg}(\bar{f}) \leq q)$. Thus $\left(x_{1}-x_{n}\right) \cdots\left(x_{n-1}-x_{n}\right) \mid g$, and therefore we can write it as $g=\left(x_{1}-x_{n}\right) \cdots\left(x_{n-1}-x_{n}\right) h$ for some polynomial $h \in R$. Since $g \in J(n, k)$ if we expand $h$ as a polynomial in $x_{n}$, its coefficients $L_{i}$ belongs to $J(n-$ $1, k-1)$. By the induction hypothesis $\operatorname{LT}\left(L_{i}\right) \in \operatorname{LT}(\rho(n-1, k-1))$. We observe that $\operatorname{LT}(h)=\operatorname{LT}\left(L_{j}\right) x_{n}^{j}$ for some $j$ and thus $\operatorname{LT}(g)=x_{1} x_{2} \cdots x_{n-1} \operatorname{LT}\left(L_{j}\right) x_{n}^{j}$. Since $\operatorname{LT}\left(L_{j}\right)$ is divisible by some element of $\operatorname{LT}(\rho(n-1, k-1))$, then $x_{1} x_{2} \cdots x_{n-1} \operatorname{LT}\left(L_{j}\right)$ is divisible by some monomial in $\operatorname{LT}(\rho(n, k))$ as desired.

If $\operatorname{LT}(g)=\operatorname{LT}(\bar{f})$ we are done. Otherwise, $\operatorname{LT}(g) \prec \operatorname{LT}(\bar{f})$ (since we use lexicographical ordering). But, in the definition of $g$ the set $S$ may be empty. In this case $\bar{f}_{S}=\bar{f}$ and we can write $g$ as

$$
g=\bar{f}+\sum_{S \neq \varnothing \text { and } S \subseteq\{1, \ldots, n-1\}}(-1)^{|S|} \bar{f}_{S} .
$$

This follows that $\operatorname{LT}(\bar{f})=\operatorname{LT}\left(\bar{f}_{S}\right)$ for a non-empty set $S \subseteq\{1, \ldots, n-1\}$. We observe that $\bar{f}_{S} \in J(r, k)$ for $n>r \geq k$ and then $\operatorname{LT}(\bar{f})=\operatorname{LT}\left(\bar{f}_{S}\right) \in \operatorname{LT}(\rho(r, k))$ by the induction hypothesis. Finally, for $n>r \geq k$, we have $\rho(r, k) \subset \rho(n, k)$, and this ends the proof of the theorem.

\section{ENUMERATING DISTINCT COLORINGS}

In this section, we correct an error in Example 3.4 in [4] to compute the number of distinct 3-colorings of the two-by-four grid graph.

In [4], De Loera has applied Theorem 1 to the general question of enumerating distinct colorings of a graph (see Lemma 3). For this, we need some definitions. Let 
us denote by $\pi(G, k)$ the number of distinct $k$-colorings of a graph $G$. Let also $P_{G}$ be the polynomial associated with the labeling of a graph $G$, i.e. if $V=\left\{x_{1}, \ldots, x_{n}\right\}$ is the set of vertices and $E(G)$ is the set of edges of $G$ then

$$
P_{G}=\prod_{i<j \text { and } x_{i} x_{j} \in E(G)}\left(x_{i}-x_{j}\right) .
$$

Now we recall the definition of the degree of an ideal. Let $R=K\left[x_{1}, \ldots, x_{n}\right]$ be a polynomial ring where $K$ is an infinite field. Let $X$ be a graded $R$-module and $\delta$ be a positive integer. We denote by $X_{\delta}$ the set of elements of $X$ of degree $\delta$. Let $I \subset R$ be a homogeneous ideal. The Hilbert series of $I$ is the power series $\operatorname{HS}_{I}(t)=\sum_{s=0}^{\infty} \operatorname{HF}_{I}(s) t^{s}$ where $\operatorname{HF}_{I}(s)$ (the Hilbert function of $I$ ) is the dimension of $(R / I)_{s}$ as an $K$-vector space.

Proposition 1. We have $\mathrm{HS}_{I}(t)=N(t) /(1-t)^{d}$ where $N(t)$ is a polynomial which is not multiple of $1-t$, and $d$ is the dimension of $I$.

For the proof of this proposition see [5], Theorem 7, Chapter 11. Now, using this proposition we could define the degree of an ideal.

Definition 1. The degree of the ideal $I$, noted by $\operatorname{deg}(I)$, is $N(1)$ where $N$ is the numerator of $\mathrm{HS}_{I}$.

We recall that the ideal $I: P_{G}^{\infty}$ is defined as

$$
I: P_{G}^{\infty}=\left\{f \in R \mid f^{m} P_{G} \in I \text { for some } m>0\right\} .
$$

Using these notations, we have the following result (see [4], Proposition 3.3).

Lemma 3. $\pi(G, k-1)=\operatorname{deg}\left(J(n, k): P_{G}^{\infty}\right)$.

Example 2. In this example, we compute the number of distinct 3-colorings of the two-by-four grid graph $H$, and we correct an error of Example 3.4 in [4] to compute it. This graph has eight vertices $x_{1}, \ldots, x_{8}$ and ten edges $x_{1} x_{2}, x_{2} x_{3}, x_{3} x_{4}, x_{4} x_{5}, x_{5} x_{6}$, $x_{6} x_{7}, x_{7} x_{8}, x_{1} x_{8}, x_{2} x_{7}, x_{3} x_{6}$. We have to compute the degree of the ideal $J(8,4)$ : $P_{H}^{\infty}$. In order to speed up the computation, De Loera has proposed to use the factorization of $P_{H}$ to compute the generators of the saturation ideals $J(8,4):\left(x_{i}-x_{j}\right)^{\infty}$ for each of the edges of $H$. He has claimed that if one computes these ten ideals, then their intersection is precisely equal to $J(8,4): P_{H}^{\infty}$ (we denote this intersection by $I$ ). But, this equality does not hold ${ }^{1}$. Using MAPLE11, we can compute $I$ and its Hilbert series where the latter is equal to

$$
\begin{aligned}
& \left(t^{13}+6 t^{12}+22 t^{11}+55 t^{10}+106 t^{9}+159 t^{8}+190 t^{7}+\right. \\
& \left.175 t^{6}+126 t^{5}+70 t^{4}+35 t^{3}+15 t^{2}+5 t+1\right) /(1-t)^{3} .
\end{aligned}
$$

\footnotetext{
${ }^{1}$ After the submission of the paper, an anonymous referee pointed out that Example 3.4 in [4] remains true if we replace "intersection" by "sum". He/She provided also a Macaulay2 code to verify this statement, see http://amirhashemi.iut.ac.ir/software.html.
} 
Therefore $\operatorname{deg}(I)=966$ which is not equal to $\pi(H, 3)$, because we will see further that the number of distinct 3-colorings of $H$ is 26 . Let us see a simple example illustrating the difference between the above ideals. Let $C_{4}$ be the 4-cycle graph with the vertices $y_{1}, \ldots, y_{4}$ and the edges $y_{1} y_{2}, y_{2} y_{3}, y_{3} y_{4}, y_{4} y_{1}$. We would like to compute $\pi\left(C_{4}, 2\right)$. We observe that $J(4,3): P_{C_{4}}^{\infty}$ is equal to $\left\langle y_{1}-y_{3}, y_{2}-y_{4}\right\rangle$, i.e. $\pi\left(C_{4}, 2\right)=1$. On the other hand,

$$
\begin{aligned}
\bigcap_{y_{i} y_{j} \in E\left(C_{4}\right)} J(4,3):\left(y_{i}-y_{j}\right)^{\infty} & =\left\langle y_{1}-y_{4}, y_{3}-y_{4}\right\rangle \cap\left\langle y_{1}-y_{2}, y_{3}-y_{4}\right\rangle \\
& \cap\left\langle y_{1}-y_{3}, y_{2}-y_{3}\right\rangle \cap\left\langle y_{1}-y_{4}, y_{2}-y_{3}\right\rangle \\
& \cap\left\langle y_{1}-y_{4}, y_{2}-y_{4}\right\rangle \cap\left\langle y_{2}-y_{4}, y_{3}-y_{4}\right\rangle \\
& \cap\left\langle y_{1}-y_{3}, y_{2}-y_{4}\right\rangle
\end{aligned}
$$

which is not equal to $J(4,3): P_{C_{4}}^{\infty}$. The Hilbert series of this intersection is equal to $\left(t^{3}+3 t^{2}+2 t+1\right) /(1-t)^{2}$, and therefore its degree is 7 .

Now, we compute $\pi(H, 3)$. Computing $J(8,4): P_{H}^{\infty}$ is not feasible in less than 12 hours (timings in this paper were conducted on a personal computer with $3.2 \mathrm{GHz}$, $2 \times \operatorname{Intel}(\mathrm{R})$-Xeon(TM) Quad core, 24 GB RAM and 64 bits under the Linux operating system). In order to speed up the computation, we use the following simple result (see [3], Theorem 11, page 196).

Lemma 4. Let $L \subset R$ be a radical ideal and $f \in R$. Let $L \cap\langle f\rangle=\left\langle g_{1}, \ldots, g_{\ell}\right\rangle$. Then $\left\{g_{1} / f, \ldots, g_{\ell} / f\right\}$ is a generating set for the ideal $L: f^{\infty}$.

Proof. It is enough to prove that any polynomial $g \in L: f^{\infty}$ belongs to $\left\langle g_{1} / f, \ldots\right.$, $\left.g_{\ell} / f\right\rangle$. We know that $g f^{m} \in L$ for some integer $m$. This follows that $(g f)^{m} \in L$, and therefore $g f \in L \cap\langle f\rangle$. Thus, $g \in\left\langle g_{1} / f, \ldots, g_{\ell} / f\right\rangle$.

We can compute $J(8,4) \cap\left\langle P_{H}\right\rangle$ and then a generating set for $J(8,4): P_{H}^{\infty}$ in 2152.549 seconds. The Hilbert series of this ideal is equal to $\left(8 t^{3}+12 t^{2}+5 t+\right.$ $1) /(1-t)^{3}$, and therefore its degree is equal to 26 .

\section{ACKNOWLEDGEMENT}

The authors would like to thank an anonymous referee for very useful comments on a previous draft.

\section{REFERENCES}

[1] N. Alon and M. Tarsi, "Colorings and orientations of graphs," Combinatorica, vol. 12, no. 2, pp. 125-134, 1992.

[2] B. Buchberger, An algorithm for finding the basis elements of the residue class ring of a zero dimensional polynomial ideal. (Ein Algorithmus zum Auffinden der Basiselemente des Restklassenringes 
nach einem nulldimensionalen Polynomideal.). Innsbruck: Univ. Innsbruck, Mathematisches Institut (Diss.), 1965.

[3] D. Cox, J. Little, and D. O'Shea, Ideals, varieties, and algorithms. An introduction to computational algebraic geometry and commutative algebra. $3 r d$ ed., ser. Undergraduate Texts in Mathematics. New York: Springer, 2007.

[4] J. A. de Loera, "Gröbner bases and graph colorings," Beitr. Algebra Geom., vol. 36, no. 1, pp. 89-96, 1995.

[5] R. Fröberg, An introduction to Gröbner bases, ser. Pure and Applied Mathematics. A WileyInterscience Series of Texts, Monographs, and Tracts. Chichester: John Wiley \& Sons, 1997.

[6] R. Lidl and H. Niederreiter, Introduction to finite fields and their applications. rev. ed. Cambridge: Univ. Press, 1994.

Authors' addresses

Amir Hashemi

Department of Mathematical Sciences,, Isfahan University of Technology, Isfahan, 84156-83111, Iran

E-mail address: Amir.Hashemi@cc.iut.ac.ir

\section{Zahra Ghaeli}

Department of Mathematical Sciences,, Isfahan University of Technology, Isfahan, 84156-83111, Iran

E-mail address: z.ghaeli@math.iut.ac.ir 